\title{
ANÁLISE DOS PROGRAMAS DE SAÚDE VINCULADOS AO SUS PARA PESSOAS COM TRANSTORNO DO ESPECTRO AUTISTA EM CURITIBA ${ }^{1}$
}

\author{
ANALYSIS OF HEALTH PROGRAMS LINKED TO SUS FOR PEOPLE WITH \\ AUTISTIC SPECTRUM DISORDER IN CURITIBA
}

Hamilton Vedovato de Marque ${ }^{2}$ Luana Ciriaco da Luz ${ }^{3}$

Cláudia Cibele Bitdinger Cobalchini ${ }^{4}$ Kátia Daniele Biscouto de Souza ${ }^{5}$

\section{RESUMO}

Desde 2012, a pessoa com Transtorno do Espectro Autista (TEA) é considerada pessoa com deficiência, passando a ter à sua disposição, no Sistema Único de Saúde (SUS), um tratamento que contemple a realidade desse indivíduo, bem como o acesso ao diagnóstico precoce. Esta pesquisa teve como objetivo caracterizar os processos de diagnóstico e tratamento ofertados pelas instituições da rede SUS, prestando atendimento especializado em Curitiba. A pesquisa envolveu análise documental e entrevistas com responsáveis das instituições de atendimento. Realizou-se a análise de prontuários de 1197 usuários com TEA dos Centros de Apoio Psicossocial - Infantil (CAPSi) e Ambulatório Enccantar entre os anos de 2015 e 2018. $\mathrm{Na}$ amostra nota-se que usuários se concentram em maior quantidade na faixa etária de 7 a 12 anos (41\%), havendo maior predomínio do sexo masculino (71\%). Cerca de 39\% dos usuários iniciaram o serviço entre 0 e 6 anos. $O$ tempo de permanência médio dos usuários nos casos em que houve alta foi de 17 meses. Em Curitiba, os atendimentos oferecidos dividiram-se em Grupo de TEA (60\%), Atendimento Individual (23\%) e Grupo de Pais (18\%) nos CAPSi e Atendimento Individual no Ambulatório Enccantar. Resultados sugerem discordância entre o previsto em documentos e o serviço prestado, e baixa permanência dos usuários no

\footnotetext{
1 A pesquisa foi realizada com o apoio de bolsa concedida pela Fundação Araucária de Apoio ao Desenvolvimento Científico e Tecnológico do Estado do Paraná.

2 Graduando em Psicologia. Bolsista pelo Programa de Bolsas de Iniciação Científica da Fundação Araucária nos anos 2017-2018. Universidade Positivo (UP), Curitiba-PR, Brasil. E-mail: vedovato.hamilton@gmail.com

3 Psicóloga graduada pela Universidade Positivo (UP), Curitiba-PR, Brasil. E-mail: luh.ciriaco@gmail.com

4 Mestre em Psicologia da Infância Adolescência, Universidade Positivo (UP), Curitiba-PR, Brasil. E-mail: claudia. cobalchini@up.edu.br

${ }^{5}$ Mestre em Análise do Comportamento. Universidade Positivo (UP), Curitiba-PR, Brasil. E-mail: katia.biscouto@up.edu.br
} 
serviço, todavia verifica-se um atendimento focado no indivíduo, com vistas à promoção da socialização. O serviço presta atendimento a um número expressivo de indivíduos, no entanto acredita-se que investimentos na capacitação dos profissionais envolvidos possam potencializar sua eficácia.

Palavras-chave: CAPS. Saúde Mental. Autismo.

\section{ABSTRACT}

Since 2012, the individual diagnosed with the Autism Spectrum Disorder (AED) has been considered someone with disabilities, having at their disposal, through the Sistema Único de Saúde (SUS), a treatment that contemplates the reality of this individual, as well as the early diagnose. This research had as its objective to distinguish the process of specialized diagnosis and treatment in the city of Curitiba. The research involved documental analysis and interviews with the staff responsible for the services, inside these institutions. Between 2015 and 2018, 1197 medical records from the Centros de Apoio Psicossocial - Infantil (CAPSi) were analyzed and it was noticed that the predominant age range is $7-12$ years old (41\%), also showing bigger predominance amongst men (71\%). Circa 39\% of the Unity of Service clients will start their treatment between the age of 0 and 6 . The permanency of the clients in the treatment is around 17 months. In Curitiba the treatment that was offered was divided in AED Group (60\%), private treatment (23\%) and also Parents Group (18\%), inside the CAPS and Enccantar Ambulatory. The results suggest disagreement between what's predicted in the documents and what's actually offered, and also low permanency of the clients in the treatment. However, it was verified that the treatment is focused on the individual, aiming at socialization promotion. The service covers a great number of individuals and it is believed that with greeted investment on the training of the professionals that will be attending at the CAPS, there will be greater efficiency in these treatments.

Keywords: CAPS. Mental Health. Autism.

\section{INTRODUÇÃO}

O Sistema Único de Saúde (SUS), instituído pela Lei Orgânica da Saúde (8080/90), é uma política pública que prevê a saúde como direito dos cidadãos e dever do Estado, viabilizando o acesso ao conjunto de serviços que atendem às necessidades de saúde da população. $O$ SUS advém com a promulgação da Constituição da República Federativa do Brasil, em 1988, quando passou a ser ofertado a todo cidadão brasileiro o acesso integral, universal e gratuito a serviços de saúde, ao longo de todo o ciclo de vida, com vistas à saúde e qualidade de vida. Sendo, portanto dever do estado tornar acessíveis seus princípios (Brasil, 2000) de atenção ao público com especificidades em sua saúde, a exemplo da atenção dedicada às 
pessoas com Transtorno do Espectro Autista (TEA), com vistas à promoção de condições de desenvolvimento de sua interação social, comunicação e autonomia.

Para uma homogeneidade no diagnóstico, em saúde mental, utiliza-se como referência o Manual Diagnóstico e Estatístico de Transtornos Mentais - DSM-V (American Psychiatric Association [APA], 2014). De acordo com este manual, o TEA integra os categorizados Transtornos do Neurodesenvolvimento, caracterizado por déficits na comunicação e interação social em diferentes contextos, as relações sociais pouco compreendidas ou inexistentes e a presença de padrões restritos e repetitivos de comportamento, interesses ou atividades. Ainda segundo o DSM-V (APA, 2014), o diagnóstico leva em consideração prejuízos na linguagem, prejuízos intelectuais, além da existência de outras condições médicas, genéticas ou ambientais.

Para uma caracterização e realização de diagnósticos destinada ao público com TEA, no Brasil, algumas leis e projetos de leis vêm sendo propostos. Uma das principais é a lei № 12.764/12, também chamada de Lei Berenice Piana, que institui a Política Nacional de Proteção aos Direitos da Pessoa com Transtorno do Espectro Autista, garantindo que a pessoa diagnosticada com TEA passe a ser considerada pessoa com deficiência para todos os efeitos legais. Sendo, portanto, seu direito o usufruto de atenção integral às suas necessidades, objetivando o diagnóstico precoce, o atendimento multiprofissional e o acesso a medicamentos e nutrientes (Brasil, 2012).

Com o intuito de regulamentar as leis que envolvam o bem-estar e o acesso das pessoas com TEA ao tratamento, em 2015, o Ministério da Saúde divulgou o documento "Linha de cuidado para a atenção às pessoas com transtornos do espectro do autismo e suas famílias na Rede de Atenção Psicossocial do Sistema Único de Saúde"por meio do qual são reforçados como os princípios básicos do SUS permeiam o cuidado à pessoa com TEA no Brasil. O documento reforça a importância e a obrigatoriedade da adoção de um Projeto Terapêutico Singular (PTS) na atenção a essa criança, que leve em consideração sua história de vida e aspectos pessoais, direcionando essa criança a um tratamento que melhor se adeque à sua realidade, de maneira que a singularidade de cada pessoa seja levada em consideração, propiciando a ampliação de seus laços sociais e suas possibilidades de circulação. Visto isso, são previstos o acesso a ferramentas terapêuticas, como: o Tratamento clínico de base psicanalítica, Análise do Comportamento Aplicada (ABA), Comunicação Suplementar Alternativa (CSA), Integração Sensorial, Tratamento e Educação para Crianças com Transtornos do Espectro do Autismo (TEACCH), Acompanhamento Terapêutico e tratamentos medicamentosos (Brasil, 2015).

Em Curitiba, à pessoa com TEA é assegurado o atendimento especializado no SUS por meio dos três Centros de Atenção Psicossocial Infantil (CAPSi Boa Vista, CAPSi Centro Vida e CAPSi Pinheirinho) e o do Ambulatório Enccantar. De acordo com a portaria № 336, de 19 de fevereiro de 2002, os CAPSi são serviços que cumprem a função de atendimento público em saúde mental as crianças e adolescentes de 0 a 18 anos com transtornos emocionais graves e/ ou transtornos decorrentes do uso de álcool e outras drogas. E o Ambulatório Enccantar, por sua vez, é um equipamento da rede SUS que oferece atendimento especializado à pessoa com TEA, dentro de Centro de Especialidades Médicas Matriz. 
Dentre as atividades de intervenção junto ao público com TEA, está o procedimento diagnóstico que se baseia na verificação da presença ou não de sinais de alerta de risco a problemas específicos, que podem estar relacionados ao espectro. Dessa forma, segundo preconiza o documento Diretrizes de Atenção à Reabilitação da Pessoa com Transtorno do Espectro Autista (Brasil, 2014), são disponibilizados no SUS instrumentos de rastreamento que podem ser aplicados por profissionais de diferentes áreas, tornando sua aplicação o mais abrangente possível. No município, a partir de 2016 estaria prevista a realização de dois procedimentos diagnósticos em crianças desde o nascimento até os 36 meses. O primeiro chama-se Indicadores Clínicos de Risco para o Desenvolvimento Infantil (IRDI), que analisa, nos 18 primeiros meses de vida, 31 requisitos da relação cuidador-bebê e o segundo é o M-Chat, um questionário composto por 23 perguntas direcionadas aos pais sobre as crianças de 18 a 36 meses, acerca de suas habilidades sociais (Curitiba, 2016).

O estudo de Lima, Couto, Solis, Oliveira e Delgado (2017) realizou uma caracterização psicossocial do público com TEA da cidade do Rio de Janeiro e os serviços utilizados por eles nos CAPSi da região. $\mathrm{O}$ estudo descritivo e quantitativo efetivou-se a partir de questionários enviados a 14 CAPSi no ano de 2011, compostos por perguntas referentes ao local de atendimento e outras questões divididas em três eixos temáticos: perfil do usuário, projeto terapêutico e dados psicossociais da clientela com TEA. Dos 13 CAPSi que responderam aos questionários, obteve-se que dos 2.213 usuários do serviço, 782 eram pessoas com TEA, predominantemente do sexo masculino (81\%) e com idades entre 10 e 19 anos. O tratamento com maior adesão envolveu atendimento semi-intensivo e com psicofármacos.

Neste cenário, na realidade de Curitiba, levantou-se a necessidade de conhecer como a rede SUS se organiza para o atendimento às crianças e adolescentes com TEA e quais instrumentos são utilizados em seu tratamento. Elegeu-se, como objetivo do estudo, a caracterização das modalidades de atendimento, ofertadas pelas instituições que compõem a rede SUS, à criança e adolescente com TEA em Curitiba. Para os objetivos específicos elencaram-se: identificar as equipes de atendimento das unidades de atendimento em saúde mental às pessoas com TEA em Curitiba; aferir a consonância entre o atendimento prestado e o que prevê o documento "Linha de cuidado para a atenção às pessoas com Transtorno do Espectro Autista e suas famílias na rede de atenção psicossocial do sistema único de saúde"; bem como identificar se os procedimentos diagnósticos condizem com o que é assegurado pela rede SUS, com intuito de conhecer os resultados gerados pela implantação dos serviços de atenção à saúde mental.

\section{PROCEDIMENTOS METODOLÓGICOS}

O estudo contemplou a análise documental, bibliográfica e a pesquisa de campo que proporcionaram a caracterização dos procedimentos e serviços prestados no período de 2015 a 2018 à população em questão, sendo esta uma pesquisa com caráter transversal, exploratório que se utiliza de abordagem qualitativa e quantitativa. Foi resultado de uma Pesquisa de Iniciação Científica realizada em uma universidade privada de Curitiba, sob o 
CAAE 69119617.7.3001.0101; cujo projeto fora aprovado pelo Comitê de Ética em Pesquisa da Universidade Positivo sob o número de parecer 2.421.173 e aprovado pelo Comitê de Ética da Secretaria Municipal de Saúde (CEP/SMS) de Curitiba sob o número de Protocolo 06/2018.

$\mathrm{Na}$ pesquisa de campo, foi conduzida uma entrevista semi-estruturada com perguntas abertas e fechadas a um representante, ou da equipe de gestores ou a um membro da equipe técnica, de cada um dos 03 CAPSi de Curitiba, e no Ambulatório Enccantar, totalizando 4 entrevistas, sendo uma delas com a presença de duas profissionais. As perguntas elaboradas foram as seguintes: 1) Quais os serviços/programas ofertados para o atendimento às pessoas com TEA?; 2) Quais os profissionais envolvidos no atendimento em saúde?; 3) Como se dão os procedimentos diagnósticos do TEA?; 4) Quais os resultados gerados pela implantação dos serviços de atenção à saúde mental às pessoas com TEA?; 5) Quais os pontos positivos da atenção à saúde das pessoas com TEA no município de Curitiba?; 6) Quais os limites e desafios ao desenvolvimento do atendimento às pessoas com TEA em Curitiba?

Os prontuários analisados foram solicitados ao gestor de cada unidade. Foram coletados separadamente os dados de pacientes ativos, ou seja, aqueles que realizavam atendimento atualmente nas unidades, e posteriormente dos pacientes inativos, que são aqueles que iniciarem o tratamento a partir de 2015, mas que por algum motivo obtiveram alta do serviço.

A análise dos prontuários teve como foco a trajetória dos pacientes dentro do serviço, desde o momento do diagnóstico até a realização do tratamento. Concluída a etapa de coleta de informações nos 3 CAPSi e no Ambulatório, essas informações foram analisadas, a fim de selecionar os dados relevantes para a pesquisa e, assim, obter descritores capazes de caracterizar o serviço.

\section{RESULTADOS E DISCUSSÃO}

A consulta aos prontuários permitiu a seleção dos dados: gênero do paciente, iniciais do nome do paciente, data de nascimento, data de inserção no serviço, origem do encaminhamento ao CAPSi, procedimento diagnóstico realizado, Projeto Terapêutico Singular (PTS) realizado, realização ou não de tratamento medicamentoso, motivos de alta, encaminhamentos realizados e data de encerramento do serviço. Não foram encontradas nos prontuários informações a respeito do procedimento diagnóstico realizado, visto que na maioria dos casos o paciente chegava ao CAPSi com encaminhamento da Unidade de Saúde. Nos casos em que o diagnóstico de TEA estava em aberto, a Unidade de Saúde direcionou o caso ao CAPSi, onde as crianças são inseridas no Grupo de TEA, e passam por Avaliação Psiquiátrica até que o diagnóstico seja realizado em conjunto com a Unidade de Saúde.

Em Curitiba, os atendimentos à pessoa com TEA concentram-se nos três Centros de Atenção Psicossocial Infantil (CAPSi) e no Ambulatório Enccantar. Os pacientes são encaminhados aos CAPSi que compõem o distrito sanitário da região na qual reside, sendo que o CAPSi Boa Vista abrange os bairros das regionais Boa Vista, Matriz e Cajuru; o CAPSi Pinheirinho abrange os bairros das regionais Boqueirão, Pinheirinho, Tatuquara e Bairro Novo e o CAPSi Centro Vida abrange os bairros das regionais Fazendinha - Portão, Santa Felicidade 
e CIC. O Ambulatório Enccantar, por sua vez, é um serviço ambulatorial com equipe específica para o atendimento a pessoas com TEA, com horário de expediente de segunda à sexta das $7 \mathrm{~h}$ às $18 \mathrm{~h}$, localizado no Centro, que realiza atendimento dos casos de TEA classificados como leve, não havendo limitação territorial para o atendimento.

As entrevistas foram realizadas com coordenadores dos serviços ou técnicos responsáveis pelos atendimentos às pessoas com TEA, designadas por um responsável do CAPSi e do Ambulatório Enccantar. Foram entrevistadas 5 profissionais envolvidas no atendimento prestado à pessoa com TEA no município, destas 2 são formadas em Psicologia, 1 em Enfermagem, 1 em Terapia Ocupacional e 1 em Serviço Social; destas entrevistadas, 3 atuavam na gestão do serviço e duas mantinham exclusivamente atividade técnica. As idades variavam entre 29 e 58 anos, com tempo de atuação na saúde entre 3 e 30 anos, e com atuação com pessoas com TEA também entre 3 e 30 anos.

Sobre o número de prontuários acessados para análise, em um dos CAPSi, não foi possível contabilizar os prontuários inativos pois a base de dados interna não contava com a identificação da Classificação Estatística Internacional de Doenças e Problemas Relacionados com a Saúde (CID), que identificaria quais seriam os pacientes com o TEA.

Também não foram analisados os prontuários que não continham informações fundamentais de identificação, como gênero do usuário ou data de início do tratamento. Sendo assim, a pesquisa contou com uma amostra de 1197 prontuários analisados, sendo desses, 205 de pacientes ativos no serviço e 992 de pacientes inativos no momento da coleta dos dados. Na tabela 1 consta a distribuição de prontuários consultados distribuídos por unidade.

Tabela 1

Relação de prontuários ativos, inativos e descartados em cada um dos locais que atendem o público com TEA em Curitiba

\begin{tabular}{|c|c|c|c|}
\hline $\begin{array}{l}\text { Unidades de } \\
\text { Atendimento }\end{array}$ & Prontuários Ativos & $\begin{array}{l}\text { Prontuários } \\
\text { Inativos }\end{array}$ & $\begin{array}{l}\text { Prontuários } \\
\text { Descartados }\end{array}$ \\
\hline CAPSi Pinheirinho & 17 & $x$ & 3 \\
\hline CAPSi Boa Vista & 8 & 47 & 12 \\
\hline CAPSi Centro Vida & 20 & 3 & $x$ \\
\hline $\begin{array}{l}\text { Ambulatório } \\
\text { Enccantar }\end{array}$ & 164 & 953 & 5 \\
\hline
\end{tabular}

Houve dificuldade na realização da coleta dos dados em decorrência da natureza dos prontuários (impressos e não informatizados), além de alguns campos de informações serem de formas diferentes registrados. Além disso, cerca de 4,49\% dos prontuários não foram encontrados pelos pesquisadores (não estavam disponíveis, apesar do registro de existência do usuário no serviço). 


\section{CARACTERIZAÇÃO GERAL DOS PACIENTES COM TEA}

Os prontuários dos atendimentos realizados entre janeiro de 2015 e maio de 2018, totalizou uma amostra de 1197 usuários inseridos nos programas da rede SUS em Curitiba; desta amostra, 205 crianças estavam em atendimento no período da realização da pesquisa, e 998 crianças que iniciaram o tratamento ou deram entrada no serviço a partir de 2015, mas que não estão mais inseridas no programa, durante o período consultado. Esses usuários se concentravam em maior quantidade na faixa etária de 7 a 12 anos (41\%), havendo maior predomínio do sexo masculino (71\%) conforme descrito na Tabela 3, fato este que corrobora com a descrição do DSM-V (APA, 2014), o qual aponta o TEA como sendo diagnosticado quatro vezes mais frequentemente no sexo masculino do que no feminino.

Dentro dessa amostra, ainda se verifica que, em $39 \%$ dos casos, o tratamento foi iniciado entre 0 a 6 anos, e outros 39\% dos casos entre 7 e 12 anos, conforme indica a Tabela 2, o que aponta que uma parcela significativa de atendimentos destina-se às crianças mais novas (117 usuários), prospectando, portanto, uma maior efetividade na realização do diagnóstico do Transtorno do Espectro Autista (TEA), na tentativa de diagnosticar o quando antes e oferecer tratamento. $\mathrm{O}$ declínio do número de usuários do programa com o passar dos anos se relaciona com a efetiva melhora do quadro do transtorno? Ou pode-se interpretar uma alta taxa de desistência dos familiares na busca de intervenções, haja vista a complexidade do tratamento e sua longa duração?

Tabela 2

Caracterização geral dos pacientes com TEA atendidos nos CAPSi da Cidade de Curitiba desde o ano de 2015

\begin{tabular}{l|r|r|r|r}
\hline Características & \multicolumn{1}{|c|}{ Ativos } & \multicolumn{1}{c|}{ Inativos } & \multicolumn{1}{c}{ Total } & \multicolumn{1}{c}{ Total (\%) } \\
\hline $\begin{array}{c}\text { Idade atual do } \\
\text { usuário }\end{array}$ & 117 & 171 & 288 & $24 \%$ \\
\hline $0-6$ anos & 73 & 418 & 491 & $41 \%$ \\
\hline $7-12$ anos & 15 & 403 & 424 & $35 \%$ \\
\hline $13-18$ anos & & & & \\
\hline $\begin{array}{c}\text { Idade do usuário } \\
\text { no início do } \\
\text { tratamento }\end{array}$ & 142 & 329 & 471 & $39 \%$ \\
\hline $0-6$ anos & 55 & 408 & 463 & $39 \%$ \\
\hline $7-12$ anos & 8 & 255 & 263 & $22 \%$ \\
\hline $13-18$ anos & 174 & 678 & 852 & $71 \%$ \\
\hline Gênero & 31 & 314 & 345 & $29 \%$ \\
\hline Masculino & & & & \\
\hline Feminino & & & & \\
\hline
\end{tabular}


Em relação aos procedimentos de diagnóstico, verificou-se que não havia, dentro dos CAPSi de Curitiba, a padronização de utilização dos instrumentos IRDI ou M-chat. Nos prontuários não constavam informações sobre a utilização de um instrumento que auxiliasse o diagnóstico. Todavia, vale lembrar que o questionário que indica os riscos de desenvolvimento psíquico está incluído na Caderneta da Criança (Brasil, 2013), que é o instrumento de acompanhamento longitudinal, ou seja, nele são registradas as variações do desenvolvimento, da criança até os seus 9 anos e 11 meses.

No Ambulatório Enccantar e nos 3 CAPSi visitados, conforme a entrevista concedida, houve a informação de que há o acolhimento dos pacientes que possuem a suspeita do TEA, encaminhados pelo Núcleo de apoio à saúde da família (NASF) ou Unidade de Saúde (US), e uma avaliação junto com a equipe multiprofissional da Unidade do CAPSi ou do Ambulatório especializado. No entanto, o diagnóstico é finalizado pelo médico. De acordo com relato da entrevistada 1: "ele vai passar em alguns momentos em alguns atendimentos pra realmente se fechar esse diagnóstico". A mesma acrescenta: "Isso é algo que ainda está sendo estruturado, porque se você analisar são várias instâncias que vão ter que ser trabalhadas. Tem uma previsão, mas não se sabe a dimensão de tudo isso, pois a partir do momento em que você ofertar isso na atenção primária, que demanda será essa? A gente não tem isso de uma forma muito precisa, isso pode ser que venha de uma forma muito grande de procura pra avaliação, pra diagnóstico e a gente ainda não sabe direcionar isso".

Diante da atualidade dos serviços destinados especificamente a este público, assim como da construção de ferramentas para diagnóstico e tratamento, evidencia-se a dificuldade em definir protocolos de atenção uniformizados.

\section{ATENDIMENTOS AOS PACIENTES COM TEA}

Dos dados obtidos, revelou-se que os pacientes têm a possibilidade de serem encaminhados para atendimento individual, atendimento em grupo, denominado Grupo TEA, Grupo de Pais ou Grupo Familiar, além da utilização de medicamentos. Estas modalidades de atenção são definidas no Projeto Terapêutico Singular, elaborado em parceria entre família e equipe, após a entrevista inicial. A seguir, na Tabela 3 observa-se a distribuição dos usuários pelas modalidades de atendimento ofertadas; vale ressaltar que para o mesmo usuário, mais de um modelo de atendimento pode ser preconizado. Não foi possível contabilizar o número de pacientes inativos do o Ambulatório Enccantar que utilizavam medicamentos. 
Tabela 3

Dados dos pacientes dos CAPSi com relação à faixa etária, tratamento e utilização de medicamentos

\begin{tabular}{|c|c|c|c|c|}
\hline $\begin{array}{l}\text { Modalidade de } \\
\text { Atendimento } \\
\text { Prestado }\end{array}$ & Ativos & Inativos & Total & Total (\%) \\
\hline $\begin{array}{l}\text { Atendimento } \\
\text { individual }\end{array}$ & 11 & 23 & 34 & $32 \%$ \\
\hline Grupo TEA & 35 & 14 & 49 & $47 \%$ \\
\hline $\begin{array}{l}\text { Grupo de pais/ } \\
\text { familiar }\end{array}$ & 11 & 11 & 22 & $21 \%$ \\
\hline $\begin{array}{l}\text { Utilizam } \\
\text { medicamentos }\end{array}$ & 28 & 14 & 42 & $52,5 \%$ \\
\hline $\begin{array}{l}\text { Não utilizam } \\
\text { medicamentos }\end{array}$ & 13 & 25 & 38 & $47,5 \%$ \\
\hline
\end{tabular}

Como apresentado na Tabela 3, a maioria dos atendimentos ocorrem na modalidade atendimento em Grupo de TEA nos CAPSi, porém deve-se salientar que, no momento em que foi realizada a coleta de dados, o atendimento individual era a única modalidade realizada no Ambulatório Enccantar. Como a maior parte dos atendimentos para o público com TEA era disponibilizado pelo Ambulatório Enccantar, 93\% dos pacientes, ativos e inativos, estavam concentrados no ambulatório, enquanto 7\% se encontravam nos CAPSi de Curitiba. Na tabela 4, constam os profissionais disponíveis no serviço e a relação do número de usuários atendidos em serviço ambulatorial de cada especialidade.

Tabela 4

Relação entre os pacientes ativos e inativos do Ambulatório Enccantar e o profissional que $o$ atendia

\begin{tabular}{l|r|r|r|r|r|r}
\hline \multicolumn{1}{c|}{ Status } & Psicologia & $\begin{array}{c}\text { Terapia } \\
\text { Ocupacional }\end{array}$ & Psicopedagogia & Fonoaudiologia & Psiquiatria & Neurologia \\
\hline Inativos & 609 & 9 & 9 & 9 & 212 & 53 \\
\hline Ativos & 86 & 47 & 0 & 48 & 9 & 0 \\
\hline
\end{tabular}

De acordo com a Tabela 4, entre os pacientes ativos $60 \%$ dos atendimentos ocorreram com o profissional da Psicologia, seguido por $20 \%$ da psiquiatria no Ambulatório Enccantar. Cada paciente pode realizar atendimentos simultâneos com os profissionais disponíveis de acordo com a necessidade apresentada. O fato sobre a ausência de registro de atendimentos em Psicopedagogia e Neurologista entre os ativos, no Ambulatório Enccantar, decorre da ausência de disponibilidade desses profissionais no serviço no momento.

Dos usuários que faziam acompanhamento em algum dos CAPSi localizados em Curitiba, 60\% estavam inseridos no Grupo TEA. Esses grupos têm por objetivo a socialização 
dos pacientes diagnosticados com TEA e são organizados de acordo com a idade deste paciente ou com o dia disponível de atendimento. Os Grupos de Pais ocorriam paralelamente ao Grupo TEA. O atendimento individual, referente a $23 \%$ dos casos, era ofertado de acordo com a necessidade do paciente.

Os grupos de pais mantinham como objetivo a troca de informações entre familiares, promovendo a partilha de experiências e espaço para que os pais expusessem suas angústias e frustrações, em relação ao cuidado. Segundo a entrevistada 3, "nesses grupos a gente orienta que as mães tenham esse tempo para ela poder dialogar, tanto com o técnico que vai estar orientando uma psicoterapia na verdade, como a relação com as mães em si" [...]"cada criança tem uma particularidade, mas no diálogo entre mães é muito enriquecedor, as mães sempre expressam isso, 'foi muito bom ter vindo aqui, hoje eu tive a oportunidade de conversar com essa mãe que tem uma diferença no modo de tratar', e assim elas vão criando um vínculo maior com o serviço, é muito bom."

Gomes, Lima, Bueno, Araújo e Souza (2015) ressaltam que, o diagnóstico do TEA gera uma série de dificuldades ao núcleo familiar, como lidar com sintomas, o acesso a serviços de saúde, relação com educação e lazer sendo, portanto, necessária a reestrutura arranjos familiares, o que por vezes, é causa de sobrecarga emocional, em especial à mãe. De acordo com Semensato, Schmidt e Bosa (2010), o relato dos pais, em relação ao cuidado com o filho com TEA, aponta a dificuldade na diferenciação de questões comportamentais ligadas ao ambiente, das relacionadas às características do TEA ou ainda dos efeitos das medicações. Scheuer (2002) sugere ainda que a própria dificuldade das pessoas com TEA em expressar suas necessidades de forma efetiva pode levar os pais a se sentirem cansados na tentativa de estabelecer uma comunicação. Verifica-se que a relevância apontada pelas entrevistas sobre o grupo de pais se dá pela possibilidade de troca e $o$ diálogo entre pares que vivenciam situações familiares semelhantes.

E em relação às atividades do Grupo de TEA, a entrevistada 3 relata: "Eles até se reconhecem, um ao outro, e tentam estabelecer alguma atividade em conjunto, mas eles não têm uma permanência nessa ação, é bem fugaz o tempo que eles ficam, e daqui a pouco eles já se voltam para eles mesmos. Mas é interessante que a gente observa: é uma atividade da criança com TEA, mas ao mesmo tempo essa forma que a gente tem de trazer eles no grupo possibilita que eles saiam disso, a gente estimula 'olha, vai com ele, faz com ele', eles se voltam muito com a gente, mas a gente faz eles voltarem para o grupo". E neste sentido o grupo tem o objetivo de atuar na potencialização da socialização, da comunicação, da adaptação aos espaços sociais frequentados pelo grupo familiar.

Nota-se que, no momento em que houve a coleta dos dados, a oferta dos serviços limitava-se às modalidades descritas, com baixa utilização das ferramentas preconizadas no documento "Linha de cuidado para a atenção às pessoas com transtornos do espectro do autismo e suas famílias na rede de atenção psicossocial do Sistema Único de Saúde" (2015), o qual prevê, como instrumentos de tratamento, a aplicação de tecnologias como: Tratamento de base clínico de base psicanalítica; Análise do Comportamento Aplicada (ABA); Comunicação Suplementar e Alternativa (CSA); Integração Sensorial; Tratamento e Educação para Crianças com TEA (TEACCH); e Acompanhamento Terapêutico. 
O documento prevê ainda, como alternativa complementar, o tratamento medicamentoso; esse recurso foi encontrado descrito nos atendimentos do município. $\mathrm{O}$ estudo permitiu identificar que a medicação é prescrita pelo profissional da Psiquiatria ou Neurologia, responsável pelo acompanhamento da criança, presente na equipe dos serviços. Nas unidades CAPSi, para 38 pacientes não houve prescrição medicamentosa, enquanto outros 42 faziam a utilização e destes, 34\%, ou seja, 27 pessoas faziam o uso da Risperidona, medicamento fornecido pelo SUS, com indicação para casos de tratamento de irritabilidade associada ao TEA. Já no Ambulatório Enccantar, 55 dos pacientes ativos faziam uso de algum medicamento, sendo que 50\% dos pacientes faziam o uso da Risperidona. Outra medicação com alta incidência de prescrição nos casos analisados é a Ritalina, que possui ação sobre impulsividade e dificuldades de atenção e concentração.

A partir dos dados coletados nos prontuários, pode-se observar que as inserções de pessoas diagnosticadas com o TEA na cidade de Curitiba nos serviços de atenção especializadas se deram em maior quantidade nos anos 2015 e 2016, com respectivamente 576 e 328 usuários inseridos. Em relação à alta, o ano de 2016 foi o com maior índice, porém a diferença entre cada ano analisado tem se mantido estável, conforme pode ser observado na Tabela 5.

Tabela 5

Relação entre quantidade de pacientes que deram início ao tratamento e os que já receberam alta

\begin{tabular}{l|r|r|r|r|r|r|r|r}
\hline \multicolumn{1}{c|}{ Gênero } & \multicolumn{4}{|c|}{ Ano de Entrada } & \multicolumn{4}{c}{ Ano de Alta } \\
\hline & 2015 & \multicolumn{1}{|c|}{2016} & 2017 & 2018 & 2015 & 2016 & \multicolumn{1}{c}{2017} & 2018 \\
\hline Masculino & 491 & 168 & 65 & 2 & 77 & 84 & 75 & 79 \\
\hline Feminino & 85 & 160 & 64 & 3 & 75 & 87 & 69 & 68 \\
\hline Total & 576 & 328 & 129 & 5 & 152 & 171 & 144 & 147 \\
\hline
\end{tabular}

Utilizou-se o ano de 2015 como parâmetro para obtenção dos dados, visto que é a partir deste ano que passou a vigorar o documento "Linha de cuidado para a atenção às pessoas com transtornos do espectro autista e suas famílias na Rede de Atenção Psicossocial do Sistema Único de Saúde". Dentro do período amostral coletado, evidenciou-se que 2015 foi o ano de maior número de novos encaminhamentos aos serviços, o que corresponde a 55\% dos que deram entrada a partir de 2015. Sugere-se que o alto número de pacientes inclusos no serviço se deva ao direcionamento de intervenções propostas pelo documento. Podendo-se observar os casos ativos e inativos, que no ano de 2016 houve um aumento significativo nos casos de alta, 28\% do total dos casos registrados.

Nos casos que receberam alta nos CAPSi, as seguintes justificativas estavam registradas: mudança de cidade (19 prontuários); transferência ou encaminhamento para outra instituição (142); abandono ou desistência (506); pedido do responsável (25); não aderência ao tratamento (14); idade (7); e alta terapêutica (207). Os outros 75 casos não estavam especificados os motivos da alta. Com isso, observa-se que, em 15\% dos casos, houve a transferência de Unidade de Saúde, passou a ser atendido por médicos conveniados a planos de saúde ou 
deram continuidade em Escolas Especiais. Pode-se perceber que a média de permanência nos tratamentos oferecidos em Curitiba é de 17 meses, sendo que a média nos CAPSi é de 6 meses e de 28 meses no Ambulatório Enccantar.

Sobre a equipe de profissionais que compõem os serviços de saúde CAPSi e ambulatório especializado em saúde mental, verificou-se que todos os equipamentos de saúde estão em acordo com as portarias ministeriais que organizam a disponibilidade dos serviços em saúde mental, contendo em sua equipe profissionais Terapeutas Ocupacionais, Psicólogos, Enfermeiros, Técnicos de Enfermagem, Médico Clínico, Médico Psiquiatra, Assistentes Sociais, Assistente Administrativo e Fonoaudiólogo, conforme a abrangência populacional do equipamento de saúde.

Segundo as entrevistadas, os desafios encontrados no atendimento seriam: a falta de qualificação dos profissionais envolvidos nos atendimentos, a falta de materiais adequados disponíveis e um local apropriado para o atendimento. É pontuado, como limitação do serviço de saúde, o fato sobre a não aceitação do diagnóstico por parte da família e os casos em que são realizados diagnósticos de Transtorno do déficit de atenção e hiperatividade (TDAH) ao invés de TEA. A Entrevistada 2 relata a este respeito: "acaba atrasando o tratamento adequado. A gente sabe que muitos médicos ainda não conseguem diagnosticar ou erra o diagnóstico, ou coloca um TDAH... ou coloca outras coisas, mas não acreditam tanto no autismo...”.

O estudo de Pondé, Novaes e Losápio (2010), realizado com 32 crianças com diagnóstico de TEA, indicou que $53,1 \%$ dos pacientes apresentaram sintomas compatíveis com o diagnóstico de TDAH, sendo que 5,8\% apresentaram sintomas que caracterizam desatenção. Com o objetivo de identificar a percepção dos pais de crianças com TEA em relação aos sintomas do TDAH, Santos e Hora (2017) realizaram entrevistas com pais de crianças e adolescentes com idade de 6 a 12 anos que tinham diagnóstico clínico de TEA, os resultados da pesquisa sinalizaram a existência de sintomas de desatenção em crianças e adolescentes autistas de acordo com a percepção dos pais.

E em relação aos pontos positivos, a entrevistada 4 mencionou "a gente tem possibilidade de um atendimento focado no território e a possibilidade de acesso a qualquer pessoa, desde a pessoa mais carente. Ele só tem que ser um usuário do SUS”. A entrevistada 5 comentou ainda "a gente já está vendo um imóvel melhor, um local adequado pra atender as crianças, nós pretendemos trabalhar muito com as famílias, isso é urgente, a gente tá sentindo essa necessidade", buscando a excelência no atendimento ao público com TEA e à família.

Com relação aos atendimentos e resultados percebidos pelas entrevistadas, apontam que a socialização é o foco dos atendimentos, como destacou a entrevistada 2: "principalmente questões da fala, questão da socialização. Crianças que tinham muita dificuldade de se relacionar, que faziam isso da forma mais rudimentar possível, a gente conseguiu, tão até na escola". Outro ponto importante foi ressaltado pela entrevistada 1: "O fato de a família se sentir acolhida e a criança já começar o tratamento logo no início do diagnóstico, faz toda a diferença”. Ou seja, a criação de repertório de socialização, de inclusão passa a ser um dos objetivos com o grupo de pais. 
As competências de interação social na pessoa com TEA estão limitadas, apresentando desde dificuldades em manter contato visual até a não habilidade em manter uma conversa com intuito de socializar (Fernandes, 2010). Práticas que promovem socialização envolvem o aprendizado de como um indivíduo deve se aproximar e interagir com o outro em diferentes meios e situações. Fernandes (2010) afirma ainda que a aprendizagem social não depende apenas de competências de comunicação verbal, mas também do entendimento, por parte do indivíduo, de pistas não verbais e decifração de regras sociais não claras. Práticas guiadas, como intervenções grupais, possibilitam ao indivíduo a aquisição de competências sociais, ao passo que à medida que a criança constrói um repertório de memórias de cenários sociais que foram vivenciados e testados na vida real, ela torna-se mais capaz em responder a situações sociais encontradas (Fernandes, 2010).

\section{CONSIDERAÇÕES FINAIS}

A caracterização do serviço dentro do período 2015-2018 pode ser realizada parcialmente: constatou-se que os equipamentos de atenção à saúde mental funcionam com a equipe mínima prevista, todavia, em relação à caracterização do serviço prestado, dados sociodemográficos do público, além de descrição de alguns instrumentos utilizados para diagnóstico e tratamento não puderam ser sistematizados, por vezes pela ausência de registro nos prontuários, outras vezes por uma diversidade de formato e conteúdo que dificulta uma categorização em critérios mais objetivos (dados de observação clínica ou de base diagnóstica partilhada pela comunidade científica). A obtenção de dados relevantes como estes conduziriam a novas análises.

Verificou-se que os procedimentos adotados nos CAPSi e Ambulatorial divergem do que é previsto no documento "Linha de cuidado para a atenção às pessoas com transtornos do espectro autista e suas famílias na Rede de Atenção Psicossocial do Sistema Único de Saúde”, ao passo que não há registro sobre a oferta dos serviços previstos (como tratamento clínico de base psicanalítica, Análise do Comportamento Aplicada (ABA), Comunicação Suplementar Alternativa (CSA), Integração Sensorial, Tratamento e Educação para Crianças com Transtornos do Espectro do Autismo (TEACCH), Acompanhamento Terapêutico), existindo entre esses apenas o tratamento medicamentoso. Em relação aos procedimentos diagnósticos, obteve-se como conclusão que o procedimento é realizado pelo médico neurologista ou psiquiatra, o qual não é especificado nos prontuários pela utilização do IRDI, M-Chat ou outros instrumentos.

Nos CAPSi e Ambulatório Enccantar os programas destinados à pessoa com TEA são baseados no acolhimento desta e de sua família, atendendo no período de 2015 a 2018, 1197 pessoas com diagnóstico de TEA. A socialização acaba sendo o ponto fundamental do tratamento e, em casos em que os profissionais vejam a necessidade, há o atendimento individual deste paciente com profissionais da psicoterapia e fonoaudiologia. Como demonstrado, 40\% dos prontuários analisados fazem utilização de medicamentos para complementar o tratamento. O serviço presta atendimento a um número expressivo de indivíduos, no entanto acredita-se 
que investimentos na capacitação dos profissionais envolvidos possam potencializar a eficácia do serviço.

O panorama aqui apresentado restringe-se ao público infantil e adolescente. Ao completar seus 18 anos, é discutido entre a família, pessoa com TEA e equipe multidisciplinar o melhor encaminhamento, podendo este ser direcionado ao CAPS TM, ou outros equipamentos da rede. Como não há uma padronização dos programas e prontuários de cada local, algumas informações não são registradas. Outra questão limitante é na obtenção de prontuários inativos, por não haver um catálogo com a identificação destes.

O serviço de acolhimento se inicia, na maioria dos casos, com as crianças entre 0 6 anos (39\%), momento importante para o início das intervenções. Em 22\% dos casos o tratamento foi iniciado entre 13 - 18 anos (22\%). Diante disso, questiona-se se o declínio da diminuição dos casos em atendimento estaria ligado a uma desistência por parte dos pais em virtude da longitudinalidade do tratamento, ou se essa diferença estaria ligada à melhora do quadro clínico dos pacientes. O tempo médio de 17 meses na permanência dos usuários que utilizam os equipamentos CAPSi e Ambulatório Enccantar, é um dado que chama atenção, visto a complexidade do TEA. Torna-se necessário o entendimento da adequação desse tempo de tratamento, relacionando ainda as questões sociais, familiares, ambientais sobre $o$ atendimento em saúde.

Pesquisas nesta área dos transtornos de desenvolvimento servem tanto à formação dos profissionais de saúde, como na avaliação sobre a efetividade das tecnologias de tratamento oferecidas na atualidade. Como forma de fomentar o desenvolvimento e aprimoramento dos serviços realizados, os pesquisadores promoveram uma Roda de Conversa nas dependências da IES, aberta ao público e que contou com a presença dos funcionários e gestores dos equipamentos em que houve a coleta dos dados e professores do curso de Psicologia; na ocasião foram apresentados os resultados obtidos na pesquisa. Na conversa foi mencionada pelos profissionais a carência de especialização ao atendimento desse público, fato esse que poderia justificar a não oferta dos serviços previstos nas diretrizes e linhas de cuidado, levantou-se ainda a dificuldade do trabalho em rede, de forma que pudesse ocorrer uma integração com as escolas a fim de que os encaminhamentos tornassem-se mais efetivos. Eventos como esse são importantes ao passo que geram trocas de experiências entre comunidade acadêmica, serviços e a sociedade.

Como este retrato é do panorama do atendimento de crianças e adolescentes com TEA em Curitiba nas instituições em funcionamento até 2018, para o presente artigo inseremse informações sobre mudanças adotadas na administração dos serviços especializados em Curitiba após a realização da pesquisa. Ainda em 2018, foi estabelecida uma entre a Prefeitura de Curitiba e o Instituto Ico-Project, para implantação do Programa da Organização Mundial de Saúde (OMS) de Treinamento de Familiares e Cuidadores de Crianças com Atrasos no Desenvolvimento, cujo objetivo era a capacitação de pais e familiares das crianças de 2 a 9 anos de idade com TEA de baixa renda, para que as famílias pudessem ser capazes de compreender necessidades e desenvolver ações diárias na atenção ao seu familiar com TEA (Curitiba, 2018). O programa dispunha de treinamento a profissionais que replicariam os conhecimentos a 
pais e cuidadores, posteriormente estender-se-ia o treinamento a profissionais da Atenção Primária de Saúde.

Em meados de 2019, a Fundação Estatal de Atenção Especializada em Saúde de Curitiba (FEAES) passou a administrar também as equipes multidisciplinares dos CAPSi Centro Vida (os outros dois já eram de sua administração). Em outubro do mesmo ano, o Ambulatório Encantar foi dividido em duas sedes, aumentando o número de profissionais, de 14 para 20 compondo a equipe de trabalho, o que ampliou sua capacidade de atendimento de 250 para 400 pessoas (Curitiba, 2019). Na sede nova, o atendimento passou a ser exclusivo para pessoas (crianças e adolescentes) com TEA, enquanto na antiga permaneceram os demais atendimentos, com foco nas vítimas de violência, dentre outras necessidades ambulatoriais do público infanto-juvenil.

No âmbito da educação e políticas de educação inclusiva a essas crianças, também em 2019 houve a inauguração do Centro de Ensino Estruturado para o Transtorno do Espectro Autista (CEETEA), onde são previstos atendimentos no contra turno escolar aos estudantes com TEA da rede municipal de ensino. O serviço também prevê o trabalho personalizado às famílias com objetivo de orientação aos cuidadores em relação ao manejo comportamental das crianças em contexto domiciliar e em relação à necessidade de atenção aos cuidados de si próprios (Curitiba, 2019).

Ainda sobre a crescente atenção ao tema, pode-se citar a constante reestruturação para atender às especificidades na área, a exemplo disso, cita-se o serviço de teleatendimento para orientação e apoio de famílias e cuidadores de pessoas com TEA que estão em isolamento domiciliar em circunstância da pandemia do Covid-19 (Curitiba, 2020).

Os resultados discutidos pela pesquisa, em consonância com as medidas recentes, ressaltam a importância da integralidade (atentando para a multidimensionalidade do desenvolvimento e da atenção) no atendimento a essa população, de forma que o cuidado e atendimento estejam sempre em consonância com as necessidades pessoais e temporais do indivíduo e da sociedade. Para pesquisas futuras, sugere-se engajamento na compreensão sobre os fatores de desistência, encaminhamento e até alta diante do quadro do TEA, assim como estudar o acompanhamento dispensado em outras unidades de atendimento (ainda na saúde, ou mesmo na educação), ou o tratamento destinado aos adultos com TEA no sistema de saúde público.

\section{REFERÊNCIAS}

Associação Americana de Psiquiatria (2014). Manual diagnóstico e estatístico de transtornos mentais (Recurso Eletrônico) (5a ed.). Porto Alegre: Artmed. Recuperado de http:// www.niip.com.br/wp-content/uploads/2018/06/Manual-Diagnosico-e-Estatistico-deTranstornos-Mentais-DSM-5-1-pdf.pdf

Brasil. Ministério da Saúde (2000). Sistema Único de Saúde (SUS): Princípios e Conquistas. Brasília: Ministério da Saúde. Recuperado de http://bvsms.saude.gov.br/bvs/publicacoes/ sus_principios.pdf 
Brasil. Ministério da Saúde (2006). Coletânea de normas para o controle social no Sistema Único de Saúde. Conselho Nacional de Saúde. Brasília: Ministério da Saúde. Recuperado de http://conselho.saude.gov.br/biblioteca/livros/coletanea_miolo.pdf

Brasil. Ministério da Saúde (2013). Caderneta de Saúde da Criança - Menino. Conselho Nacional de saúde. Recuperado de http://bvsms.saude.gov.br/bvs/publicacoes/ caderneta_saude_crianca_menino.pdf

Brasil. Ministério da Saúde (2014). Diretrizes de Atenção à Reabilitação da Pessoa com Transtorno do Espectro do Autismo. Brasília: Ministério da Saúde. Recuperado de http:// bvsms.saude.gov.br/bvs/publicacoes/diretrizes_atencao_reabilitacao_pessoa_autismo.pdf

Brasil. Ministério da Saúde (2015). Linha de cuidado para a atenção às pessoas com transtornos do espectro autista e suas famílias na Rede de Atenção Psicossocial do Sistema Único de Saúde. Brasília: Ministério da Saúde. Recuperado de http://bvsms. saude.gov.br/bvs/publicacoes/linha_cuidado_atencao_pessoas_transtorno.pdf

Curitiba (2018). Em parceria pioneira com a OMS, Curitiba apoia famílias de crianças com autismo. Prefeitura Municipal de Curitiba. Recuperado de https://www.curitiba.pr.gov. br/noticias/em-parceria-pioneira-com-a-oms-curitiba-apoia-familias-de-criancas-comautismo/45153

Curitiba (2019a). Prefeitura inaugura nova sede do Ambulatório Encantar, no Alto da Glória. Prefeitura Municipal de Curitiba. Recuperado de https://www.curitiba.pr.gov.br/noticias/ prefeitura-inaugura-nova-sede-do-ambulatorio-encantar-no-alto-da-gloria/53113

Curitiba (2019b). Estudantes com Autismo ganham Centro de Atendimento-Educacional. Prefeitura Municipal de Curitiba. Recuperado de https://www.curitiba.pr.gov.br/noticias/ estudantes-com-autismo-ganham-centro-de-atendimento-educacional/52616

Curitiba (2020) Saúde. Saúde lança teleatendimento para apoio às famílias de autistas. Prefeitura Municipal de Curitiba. Recuperado de https://www.curitiba.pr.gov.br/noticias/ saude-lanca-teleatendimento-para-apoio-as-familias-de-autistas/55676

Decreto-Portaria n. 336, de 19 de fevereiro de 2002. Dispõe sobre a proteção e os direitos das pessoas portadoras de transtornos mentais e redireciona o modelo assistencial em saúde mental. Recuperado de http://bvsms.saude.gov.br/bvs/saudelegis/gm/2002/ prt0336_19_02_2002.html

Fernandes, S. (2010). A adequabilidade do modelo teacch para a promoção do desenvolvimento da criança com autismo (Dissertação de Mestrado). Escola Superior de Educação de Paula Frassinetti, Porto, Portugal. Recuperado de http://repositorio.esepf.pt/ jspui/bitstream/20.500.11796/796/2/PG-EE-2010_SalomeFernandes.pdf

Gomes, P. H. L., Lima, L. H. L., Bueno, M. K. G., Araújo, L. A. A., Souza, N. M (2015). Autism in Brazil: a systematic review of family challenges and coping. Jornal de Pediatria (Versão em Português). 91(2), 111-121. Recuperado de: https://www.sciencedirect.com/science/ article/pii/S2255553615000221

Lima, R. C., Couto, M. C. V., Solis, F. P., Oliveira, B. D. C., \& Delgado, P. G. G. (2017). Atenção psicossocial a crianças e adolescentes com autismo nos CAPSi da região metropolitana do Rio de Janeiro. Revista Saúde e Sociedade, 26(1), 196-207. Recuperado de http:// www.scielo.br/pdf/sausoc/v26n1/1984-0470-sausoc-26-01-00196.pdf 
Pondé, M. P., Novaes, C. M., \& Losápio, M. F. (2010). Frequência de sintomas de transtorno de déficit de atenção e hiperatividade em crianças autistas. Arq. Neuro-Psiquiatr. [online], 68(1), 103-106. DOI: https://doi.org/10.1590/S0004-282X2010000100022

Santos, E. D. M., \& Hora, A. F. L. T. (2017). Percepção dos pais sobre os sintomas do TDAH em crianças e adolescentes diagnosticados com TEA. Ceuma Perspectivas, 30(01), 25255576. 2017. DOI: https://doi.org/10.24863/rccp.v30i2.107

Scheuer, C. (2002). Distúrbios da linguagem nos transtornos invasivos do comportamento. In C. R. Baptista, \& C. Bosa (Orgs.), Autismo e educação: reflexões e propostas de intervenção (pp. 51-62). Porto Alegre: Artmed.

Semensato M. R., Schmidt, C., \& Bosa, C. A. (2010) Grupo de familiares de pessoas com autismo: relatos de experiências parentais. Aletheia, (32), 183-194. Recuperado de http://www.periodicos.ulbra.br/index.php/aletheia/article/view/3532

Recebido em: 22-04-2020

Primeira decisão editorial: 21-05-2020

Aceito em: 04-06-2020 